\title{
A NEW METHOD FOR DETERMINING THE REACTIONS OF MECHANICAL CONSTRAINTS
}

\author{
Do SanH, DO Dang KHOA \\ Hanoi University of Technology
}

\begin{abstract}
In the present paper it is introduced the method for determining the reactions of mechanical constraints ( holonomic and nonholonomic constraints).

As is known, for studying dynamical characters of a mechanical system it is necessary to determine the constraint reactions acting on the system. Up to now, the reactions are calculated through Lagrange's multipliers. By such a way the reactions are determined only indirectly. In the $[3,4]$, two methods of determining directly the reactions are discussed. However, for applying these methods, it is necessary to compute the inverse matrix of the matrix of inertia. This thing in general is not convenient, specially when the matrix of inertial is of large size and dense.

In the present paper it is represented the method for determining the constraint reactions, by which it is possible to avoid inertia the computation of the inverse matrix of the matrix of inertia is avoided. For this in the paper it is used the middle variables by which we obtain a closed set of algebraic equations for directly determining reactions.
\end{abstract}

\section{INTRODUCTION}

In the development of technics the completed systems are very interested. In accorsance with this, the dynamic study of constrained systems is becoming more and more c....rtant.

As is known, up to now there is not yet a general method for determining directly - - teactions of constraints. There is only the method of Lagrange's multipliers, by which $-\approx$ zeactions are determined through Lagrange's multipliers [ 2, 7 ]. Besides this, there $=t \ldots$ of methods of calculating the reactions, which are discussed in the works[ 3,4$]$. F....:er, in order to apply the last methods, it is necessary to compute the inverse matrix atrix of inertia. This thing is not always convenient, for example, when the matrix - $=-\mathrm{rti}$ is of large size and is dense.

In present paper it is constructed the method, which overcomes the foible of two above z--:-ined methods.

\section{THE METHOD FOR DETERMINING THE REACTIONS OF CONSTRAINTS}

Let us consider the system with generalised coordinates $q_{j}(j=\overline{1}, m)$. The expression $\therefore-$ tinetic and potential energy of the system under consideration are of the form:

$$
T=\frac{1}{2} \sum_{i, j=1}^{m} a_{i j} \dot{q}_{i} \dot{q}_{j} ; \prod=\prod\left(q_{1}, q_{2}, \ldots, q_{m}\right)
$$


Suppose that the system subjected to stationary constraints of the form:

$$
\sum_{j=1}^{m} b_{\alpha j} \ddot{q}_{j}+b_{\alpha}=0 ; \quad \alpha=\overline{1, r}
$$

where the coefficients $b_{\alpha j}, \mathrm{~b}_{\alpha}$ are the continue functions of coordinates and velocities, $\mathrm{i}$. e,

$$
\begin{aligned}
& b_{\alpha j}=b_{\alpha j}\left(q_{1}, q_{2}, \ldots, q_{m}, \dot{q}_{1}, \dot{q}_{2}, \ldots, \dot{q}_{m}\right), \\
& b_{\alpha}=b_{\alpha}\left(q_{1}, q_{2}, \ldots, q_{m}, \dot{q}_{1}, \dot{q}_{2}, \ldots, \dot{q}_{m}\right) .
\end{aligned}
$$

It is noticed that, the coefficients $a_{i j}(i, j=\overline{1, m})$ in the expression of kinetic energy are the functions of coordinates, i. e.

$$
a_{i j}=a_{i j}\left(q_{1}, q_{2}, \ldots, q_{m}\right) .
$$

It is easy to show, that the holonomic constraints, linear and nonlinear nonholonomic constraints with respect to the velocitees are written in the form (2. 2) too.

Let us denote the generalised force corresponding to the coordinate $q_{j}$ by $Q_{j}$, which is the function of coordinates and velocities, i. e., $Q_{j}=Q_{j}\left(q_{1}, q_{2}, \ldots, q_{m}, \dot{q}_{1}, \dot{q}_{2}, \ldots, \dot{q}_{m}\right)$.

For compact, let us introduce the following matrix notations:

$$
\begin{aligned}
\mathbf{q}^{T} & =\left\|q_{1}, q_{2}, \ldots, q_{m}\right\|, \\
\dot{\mathbf{q}}^{T} & =\left\|\dot{q}_{1}, \dot{q}_{2}, \ldots, \dot{q}_{m}\right\|, \\
\ddot{\mathbf{q}}^{T} & =\left\|\ddot{q}_{1}, \ddot{q}_{2}, \ldots, \ddot{q}_{m}\right\| .
\end{aligned}
$$

These are the $(m \times 1)$ matries of coordinates, velocities and accelerations respectively. The symbol " $T$ " is notation of transpose of matrix.

the $\mathbf{Q}$ is the $(m \times 1)$ matrix of generalised forces,

$$
\mathbf{Q}^{T}=\left\|Q_{1}, Q_{2}, \ldots, Q_{m}\right\| .
$$

In the matrix form the expression of the kinetic energy will be written as follows

$$
T=\frac{1}{2} \dot{\mathbf{q}}^{\mathbf{T}} \mathbf{A} \dot{\mathbf{q}}
$$

where $\mathbf{A}$ is an $(m \times m)$ matrix of inertia of the system.

the equations of constraints will be

$$
\mathbf{b} \ddot{\mathbf{q}}+\mathbf{b}_{\mathbf{0}}=\mathbf{0},
$$

where $\mathbf{b}$ and $\mathbf{b}_{0}$ are the $(r \times m)$ and $(r \times 1)$ matrices respectively. The their elements are the functions of generalised coordinates and velocities.

As is known [3,4] the equations of motion of the system can be written as follows:

$$
\frac{d}{d t} \frac{\partial T}{\partial \dot{\mathbf{q}}}-\frac{\partial T}{\partial \mathbf{q}}=\mathbf{Q}+\mathbf{R},
$$


where $\mathbf{R}$ is the matrix of the reactions of the constraints (2. 3) acting on the system:

$$
\mathbf{R}^{\mathrm{T}}=\left\|R_{1}, R_{2}, \ldots, R_{m}\right\| .
$$

By the Principle of Compatibility [3,4] the reactions must satisfy the algebraic equations, which in the matrix form are written as follows

$$
\mathbf{B R}+\mathbf{B Q}^{*}+\mathbf{b}_{\mathbf{0}}=\mathbf{0}
$$

where

$$
\begin{gathered}
\mathbf{B}=\mathbf{b} \mathbf{A}^{-\mathbf{1}} \\
\mathbf{Q}^{*}=\mathbf{Q}+\mathbf{A} \Psi .
\end{gathered}
$$

The $(m \times 1)$ matrix $\boldsymbol{\Psi}$ has the elements

$$
\psi_{j}=\sum_{k, s=1}^{m}\left(\frac{\partial a_{j s}}{\partial q_{k}}+\frac{\partial a_{k s}}{\partial q_{j}}-\frac{\partial a_{j k}}{\partial q_{s}}\right) \dot{q}_{k} \dot{q}_{s}
$$

where the $a_{i j}(i, j=\overline{1, m})$ are the elements of the $(m \times m)$ matrix of inertia, but $\mathbf{A}^{-1}$ is the inverse matrix of the last.

By such a way we obtain $r$ algebraic equations containing $m$ unknowns $R_{j}(r<m)$. In order to determine the reactions $R_{j}(j=\overline{1, m})$ it is necessary to fill up $k=(m-r)$ equations containing only $m$ unknowns $R_{j}(j=\overline{1, m})$, which in addition to $m$ equations (2.5) yield a complete set of equations of unknowns $R_{j}(j=\overline{1, m})$

For this purpose let us to use the condition of ideality of the constraints (2.2). This condition in the matrix form is written as follows [3]

$$
\mathbf{D R}=\mathbf{0}
$$

where the $(k \times m)$ matrix $\mathbf{D}$ consists of the elements, which are the coefficients in term of the expressions of generalised accelerations represented through independent generalised accelerations.

The equations ( 2.5 ) and ( 2.9 ) yield a closed set of $m$ algebraic equations containing $m$ unkowns $R_{j}(j=\overline{1, m})$. By solving it we get the reactions of the constraints (2.2), which are the functions of coordinates and velocities, that is:

$$
\mathbf{R}=\mathbf{R}(\mathbf{q}, \dot{\mathbf{q}})
$$

Equations of motion of the system with the constraints will be written in the form:

$$
\frac{d}{d t}\left(\frac{\partial T}{\partial \dot{q}}\right)-\frac{\partial T}{\partial q}=\mathbf{Q}+\mathbf{R} .
$$

Of course, these equations take an important part in dynamic study of mechanical systems with constraints.

However, in order to write the equations ( 2.5) and (2.9) it is necessary to compute the inverse matrix of the matrix of inertia A. This thing is unconvenient when the matrix 
$\mathbf{A}$ is of large size and is dense, for example, in the case of the elements of $\mathbf{A}$ being the trigonometry functions.

In order to overcome this let us introduce in consideration the middle unknowns $R_{j}^{o}(j=$ $\overline{1, m})$, which satisfy the following relation

$$
\mathbf{R}^{\mathbf{0}}=\mathbf{A}^{-1} \mathbf{R}
$$

where $\mathbf{R}^{0}$ the $(m \times 1)$ matrix of elements $R_{j}^{o}(j=\overline{1, m})$ but $\mathbf{R}$ - the $(m \times 1)$ matrix of reactions of the constraints (2.2) and $\mathbf{A}^{-1}$ - the inverse matrix of the matrix of inertia A.

By the tranformation (2.12) the equation (2.5) will take the form

$$
\mathbf{b R}^{0}+\mathbf{b Q}^{0}+\mathbf{b}_{0}=\mathbf{0}
$$

but the (2.9) will be now

$$
\mathbf{D}^{0} \mathbf{R}^{0}=0 .
$$

The new unknown $\mathbf{Q}^{0}$ being the $(m \times 1)$ matrix will satisfy the following equation:

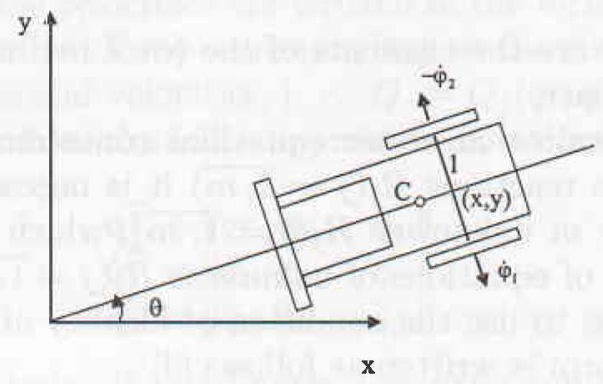

Fig. 1

$$
\mathbf{A} \mathbf{Q}^{0}=\mathbf{Q}^{*}
$$

where $\mathbf{A}$ is the matrix of inertia, but $\mathbf{Q}^{*}$ and $\mathbf{D}^{\mathbf{0}}$ are computed respectively by the relation (2.7) and

$$
\mathbf{D}^{\mathbf{0}}=\mathbf{D A}
$$

with $\mathrm{D}$ is constructed as in (2.9).

It is noticed that, the matrice $\mathbf{b}$ and $\mathbf{b}_{0}$ are described in (2.3).

In sum we obtain $2 m$ equations (2.13), (2.14) and (2.15) contain $2 m$ unknowns $R_{i}^{0}$ and $Q_{i}^{0}(i=\overline{1, m})$

By solving these equations we obtain $\mathbf{R}^{0}$ and further by (2.12) we get reactions of the constraints $(2.2)$, that is

$$
\mathbf{R}=\mathbf{A} \mathbf{R}^{\mathbf{0}}
$$

By such a way the reactions are calculated. The represented computation scheme permits to evade the calculation of the inverse matrix of the matrix of inertia.

It is noticed, that in the case of holonomic constraints or nonholonomic constraints linear or nonlinear with respect to velocities the constraints equations must be treated as the first integrals of the equations of motion (2.11) 
It is easy to show that in such a case we have $\mathbf{R}(\mathbf{q}, \mathbf{0})=0$. This means that at the equilibrium position the reactions are equal to zero.

For illustrating let us consider the two wheeled carriage (Fig.1) on a rough horizontal plane [7]. The position of the system is determined by five generalised coordinates.

Let us assume that the position of the body is defined by the coordinates $x, y, \theta, \varphi_{1}$ and $\varphi_{2}$ where $x, y$ are the coordinates of the point of intersection of the symmetry axis of the carriage with the axle on which the wheels are mounted; $\theta$ - the angle between the symmetry axis of the carriage and the $O x$-axis, $\varphi_{1}$ and $\varphi_{2}$ - the angles of rotation of the left-hand wheel and the right -hand wheel respectively. We shall assume that the body of the carriage does not undergo vertical displacements. The condition of rolling without sliding lead to three kinematic constraints ( $a$ is the radius of the wheels and $b$ if half the length of the axle):

For the left-hand wheel:

i.

$$
\dot{x} \cos \theta+\dot{y} \sin \theta-b \dot{\theta}+a \dot{\varphi}_{2}=0 .
$$

For the right-hand wheel:

$$
\dot{x} \cos \theta+\dot{y} \sin \theta+b \dot{\theta}+a \dot{\varphi}_{1}=0 .
$$

The absence of lateral sliding of both wheel yields

$$
\dot{x} \sin \theta-\dot{y} \cos \theta=0 \text {. }
$$

Then the system under consideration has two degrees of freedom.

Let us denote the mass centre of the body by $C_{0}$, which is situated on the symmetry axis at a distance $L$ from the point $(x, y)$.

The kinetic energy of the carriage can be written as follows

F

$$
T=\frac{1}{2} m\left(\dot{x}^{2}+\dot{y}^{2}\right)+\frac{1}{2} J \dot{\theta}^{2}+\frac{1}{2} C\left(\dot{\varphi}_{1}^{2}+\dot{\varphi}_{2}^{2}\right)+m_{0} L \dot{\theta}(\dot{y} \cos \theta-\dot{x} \sin \theta) .
$$

where $\boldsymbol{m}=m_{0}+2 m_{1}, m$ is the mass of the whole carriage, $m_{0}$ - the mass of the body, $m_{1}$ - the mass of each wheel (the wheels are assumed to be identical ), $J=m_{0} k_{0}^{2}+2 m b^{2}+2 A$ - the moment of inertia of the carriage when it rotates as a whole about the vertical axis passing through the point $C(x, y), k_{0}$ - the radius of gyration of the body about the vertical passing through $C(x, y), A$-the moment of inertia of a wheel about a diameter, $C$-the axial moment; of inertia.

The potential energy is equal to zero, i.e. $\Pi=0$.

Let us choose the independent coordinates to be $\mathrm{x}$ and $\theta$.

By the constraint equations (a) (b) and (c) we obtain:

$$
\dot{y}=\operatorname{tg} \theta \dot{x}, \quad \dot{\varphi}_{1}=-\frac{1}{a \cos \theta} \dot{x}-\frac{b}{a} \dot{\theta} ; \quad \dot{\varphi}_{2}=-\frac{1}{a \cos \theta} \dot{x}+\frac{b}{a} \dot{\theta} .
$$

The $(2 \times 5)$ matrix $\mathbf{D}$ in (2.9) in accordance with the relations (e) is of the form now

$$
\mathbf{D}=\left[\begin{array}{ccccc}
1 & \operatorname{tg} \theta & 0 & -\frac{1}{a \cos \theta} & -\frac{1}{a \cos \theta} \\
0 & 0 & 1 & -\frac{b}{a} & \frac{b}{a}
\end{array}\right] .
$$


The $(5 \times 5)$ matrix of inertia of the system will be

$$
\mathbf{A}=\left[\begin{array}{ccccc}
m & 0 & -m_{0} L \sin \theta & 0 & 0 \\
0 & m & -m_{0} L \cos \theta & 0 & 0 \\
-m_{0} L \sin \theta & m_{0} L \cos \theta & J & 0 & 0 \\
0 & 0 & 0 & C & 0 \\
0 & 0 & 0 & 0 & 0
\end{array}\right] .
$$

In accordance with $(2.16)$ the $(2 \times 5)$ matrix $\mathrm{D}^{0}$ has the form

$$
\mathbf{D}^{\mathbf{0}}=\mathbf{D A}=\left[\begin{array}{ccccc}
m & m \operatorname{tg} \theta & 0 & -\frac{C}{a \cos \theta} & -\frac{C}{a \cos \theta} \\
-m_{0} L \sin \theta & m_{0} L \sin \theta & J & -\frac{c b}{a} & \frac{c b}{a}
\end{array}\right] .
$$

By the constraint equations (a) (b) and (c) we obtain the $(3 \times 5)$ matrix $\mathbf{b}$ and the $(3 \times 1)$ matrix $\mathbf{b}_{\mathbf{0}}$, they are

$$
\begin{gathered}
\mathbf{b}=\left[\begin{array}{lllll}
\cos \theta & \sin \theta & b & a & 0 \\
\cos \theta & \sin \theta & b & 0 & a \\
\sin \theta & \cos \theta & 0 & 0 & 0
\end{array}\right], \\
\mathbf{b}_{0}^{\mathbf{T}}=\left[\begin{array}{lll}
0 & 0 & -(\cos \theta \dot{x}+\sin \theta \dot{y}) \dot{\theta}
\end{array}\right] .
\end{gathered}
$$

Because of the potential energy being to zero and the absence of external forces, the matrix of generalised force is equal to zero, i.e. $\mathbf{Q}=0$.

It is easy to calculate the second term in (2.7), which is a matrix $(m \times 1)$ and has the following form:

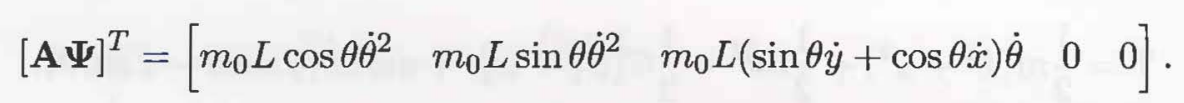

The $(5 \times 1)$ matrix $\mathbf{Q}^{*}$ will be

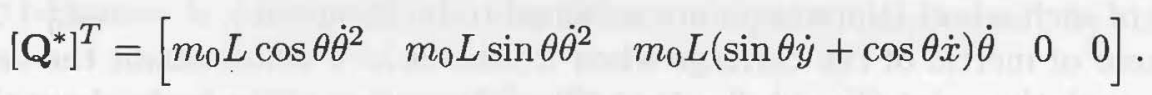

The equations (2.13), (2.14), (2.15) now will be written as follows:

$$
\begin{aligned}
& \cos \theta R_{x}^{0}+\sin \theta R_{y}^{0}+b R_{\theta}^{0}+a R_{\varphi_{1}}^{0}+\cos \theta Q_{x}^{0}+\sin \theta Q_{y}^{0}+b Q_{\theta}^{0}=0, \\
& \cos \theta R_{x}^{0}+\sin \theta R_{y}^{0}-b R_{\theta}^{0}+a R_{\varphi_{1}}^{0}+\cos \theta Q_{x}^{0}+\sin \theta Q_{y}^{0}-b Q_{\theta}^{0}=0, \\
& \sin \theta R_{x}^{0}-\cos \theta R_{y}^{0}+\sin \theta Q_{x}^{0}-\cos \theta Q_{y}^{0}+(\cos \theta \dot{x}+\sin \theta \dot{y}) \dot{\theta}=0, \\
& m R_{x}^{0}-m t g \theta R_{y}^{0}+\sin \theta Q_{x}^{0}-\cos \theta Q_{y}^{0}-(\cos \theta \dot{x}+\sin \theta \dot{y}) \dot{\theta}=0, \\
& m R_{x}^{0}-m t g \theta R_{y}^{0}-\frac{C}{a \sin \theta} R_{\varphi_{1}}-\frac{C}{a \cos \theta} R_{\varphi_{2}}, \\
& m Q_{x}^{0}-m_{0} L \sin \theta Q_{\theta}^{0}=m_{0} L \cos \theta \dot{\theta}^{2}, \\
& m Q_{y}^{0}+m_{0} L \cos \theta Q_{\theta}^{0}=m_{0} L \sin \theta \dot{\theta}^{2}, \\
& -m_{0} L \sin \theta Q_{x}^{0}+m_{0} L \cos \theta Q_{y}^{0}+J Q_{\theta}^{0}=m_{0} L(\cos \theta \dot{x}+\sin \theta \dot{y}) \dot{\theta} .
\end{aligned}
$$


Solving these equations we get

$$
\begin{aligned}
& R_{x}^{0}=-\left[\frac{m J}{m J-m_{0}^{2} L^{2}} \operatorname{tg} \theta \dot{x} \dot{\theta}+\frac{m_{0}}{m} L^{2} \frac{\cos \theta}{\left(1+\frac{m a^{2}}{2 C}\right)} \dot{\theta}^{2}\right] \\
& R_{y}^{0}=\frac{m J}{m J-m_{0}^{2} L^{2}} \operatorname{tg} \theta \dot{x} \dot{\theta}-\frac{m_{0}}{m} L^{2} \frac{\cos \theta}{\left(1+\frac{m a^{2}}{2 C}\right)} \dot{\theta}^{2}, \\
& R_{\theta}^{0}=-\frac{m m_{0} L}{\cos \theta\left(m J-m_{0}^{2} L^{2}\right)} \dot{x} \dot{\theta} \\
& \boldsymbol{R}_{\varphi_{1}}^{0}=\boldsymbol{R}_{\varphi_{2}}^{0}=-\frac{a}{2 C} m_{0} L \frac{1}{1+\frac{m a^{2}}{2 C}} \dot{\theta}^{2} .
\end{aligned}
$$

By the formulas (2.17) it is easy to compute the reactions of the constraints (a) (b) and (c) acting on the carriage. They are:

$$
\begin{aligned}
& R_{c}=m R_{x}^{0}-m_{0} L \sin \theta R_{\theta}^{0}=-m \operatorname{tg} \theta \dot{x} \dot{\theta}-\frac{m_{0} L^{2}}{1+\frac{m a^{2}}{2 C}} \cos \theta \dot{\theta}^{2}, \\
& R_{y}=m R_{y}^{0}+m_{0} L \sin \theta \dot{R}_{\theta}^{0}=m \dot{x} \dot{\theta}-\frac{m L^{2}}{1+\frac{m a^{2}}{2 C}} \sin \theta \dot{\theta}^{2}, \\
& R_{\theta}=-m_{0} L \sin \theta R_{x}^{0}+m_{0} L \cos \theta R_{y}^{0}+J R_{\theta}^{0}=0, \\
& R_{\varphi_{1}}=R_{\varphi_{2}}=-c R_{\varphi_{1}}^{0}=-\frac{a}{2} m_{0} L^{2} \frac{1}{1+\frac{m a^{2}}{2 C}} \dot{\theta}^{2} .
\end{aligned}
$$

Equations of motion of the carriage now will be written as follows:

$$
\begin{aligned}
& m \ddot{x}-m_{0} L \sin \theta \ddot{\theta}=m_{0} L \cos \theta \dot{\theta}^{2}-m \operatorname{tg} \theta \dot{x} \dot{\theta}-\frac{m_{0} L^{2}}{1+\frac{m a^{2}}{2 C}} \cos \theta \dot{\theta}^{2}, \\
& \dot{A r}_{4}, m_{0} L \sin \theta \ddot{\theta}=m_{0} L \sin \theta \dot{\theta}^{2}-m \dot{x} \dot{\theta}-\frac{m_{0} L^{2}}{1+\frac{m a^{2}}{2 C}} \sin \theta \dot{\theta}^{2}, \\
& C \ddot{\varphi}_{1}=-\frac{a}{2} m_{0} L^{2} \frac{1}{1+\frac{m a^{2}}{2 c}} \dot{\theta}^{2}, \\
& C \ddot{\varphi}_{2}=-\frac{a}{2} m_{0} L^{2} \frac{1}{1+\frac{m a^{2}}{2 C}} \dot{\theta}^{2} .
\end{aligned}
$$

It is cleary that the application of the other methods for this problem is very difficulty to get these results.

\section{CONCLUSION}

The dynamic investigation of constrained systems is very important, but this is a hard problem because of the determining the reactions of constraints on the system. 
As is known, up to the present time, there is not yet any method for solving usefully above mentioned problem.

In the present paper it is represented the method, which permits to compute directly the reactions of constraints and therefore the dynamic study of the system will be more easy.

This publication is completed with financial support from the National Basic Research Program in Natural Sciences.

\section{REFERENCES}

1. P. Appell, Traite de Mecanique Rationnelle, Paris, t.II, 1953.

2. Dobronravov v.v., Bases of Nonholonomic Mechanics, Vysha Skola, Moscow 1976 (in Russian).

3. Do Sanh, On the motion of constrained system, The Thesis of Doctor of Science, Hanoi University of Technology, Hanoi, 1984 (in Vietnamese)

4. Do Sanh, On the Principle of Compatibility and the Equation of a Constrained Mechamical System, ZAMM, 1980

5. Do Sanh, Dinh Van Phong, Do Dang Khoa, Problem of optimal control of program motion of mechanical system, Proceedings of the $8^{\text {th }}$ International Conference on Mechatronies Technology, ICMT 2004, November 8-12, 2004, Hanoi, Vietnam

6. A. I. Lurie, Analytical Mechanics, Publ. Fizmatgiz, 1961 (in Russian)

7. Ju. I. Neimark and N. A. Fufaev, Dynamics of Nonholonomic System, Publ "Nauka", 1967 (in Russian).

Received October 4, 2005

Revised November 1, 2005

\section{MộT PHƯƠNG PHÁP MỚI ĐỂ XÁC ĐỊNH CÁC PHẢN LỰC CƯA CÁC LIÊN KẾT LÊN CÂC Hิ̣̂ CƠ HỌC}

Trong bài báo đã đề xuất một phương pháp cho việc xác định trực tiếp các phản lực liên kết lên các hệ cơ học, cho đến nay bài toán này chưa được giải quyết một cách có hiệu quá do chỉ sử dụng phương trình chuyển động dạng nhân từ. Phương pháp nêu ra đã cho phép xác định trực tiếp các phán lực liên kết và nhờ đó có thể xây dựng phương trình chuyển động của cơ hệ không chứa các nhân tử Lagrange. 